\title{
ON THE ADAMS CONJECTURE
}

\author{
I. DIBAG
}

\begin{abstract}
We give a short elementary and self-contained proof to the Adams conjecture. We then present three equivalent formulations of the conjecture.
\end{abstract}

0. Introduction. The purpose of this note is to give a short elementary and self-contained proof to the Adams conjecture $[10,11]$, in particular assuming only Dold's Theorem $\bmod k$. Our argument is a variation, using the elementary notion of degree functions, on the Becker-Gottlieb proof [6] which has the advantage of making no appeal to infinite loop space structures on $S G$ and $B S G$.

We define a nondegenerate form $q$ called the degree function on the group $K F^{\mathrm{or}}(B)$ of stable fibre homotopy classes of orientable spherical Hurewicz fibrings over $B$. The crucial point in our argument is Proposition 1.5.1 which states that if $p: E \rightarrow B$ is a fibre bundle with fibre $F$ then $p^{!}: \widetilde{K F}^{\text {or }}(B) \rightarrow \widetilde{K F}^{\text {or }}(E)$ satisfies $q(u) \mid \chi(F) q\left(p^{!}(u)\right), u \in \widetilde{K F}^{\text {or }}(B)$ where $\chi(F)$ is the Euler-Poincaré characteristic of $F$. This enables us to extend the proof of the Adams conjecture from $N(T)$ to $S O(2 n)$-bundles where $N(T)$ is the normalizer of the maximal torus $T$ in $S O(2 n)$. We also eliminate the inessential use in [6] of the transfer for finite coverings.

In $\S 2$ we present three alternative formulations of the Adams conjecture and prove their equivalence. Of these the third one gives more information than the usual statement of the conjecture.

1.1. The fibrewise map $f_{k}(\xi): E_{\xi} \rightarrow E_{\psi} k_{(\xi)}$, for $N(T)$-bundles. Let $V=R^{2 n}$ be the $N(T)$-module obtained by restricting the usual $S O(2 n)$-action to $N(T)$. Decompose $V=V_{1} \oplus \cdots \oplus V_{n}$ where each $V_{i}$ is isomorphic to $R^{2}$ and is spanned by basis elements $e_{2 i-1}, e_{2 i}$. The permutation group $S_{n}$ acts on $V$ by permuting the factors $V_{i}$. Let $u_{i} \in O(2 n)$ permute the basis elements $e_{2 i-1}$ and $e_{2 i}$ and leave $V_{j}$ for $j \neq i$ pointwise fixed. Products of an even number of $u_{i}$ 's form a subgroup $U_{n}$ of $S O(2 n)$ of order $2^{n-1}$. Let $W_{n}=S_{n} \times U_{n}$ be their twisted cross product which is a subgroup of $S O(2 n)$ of order $2^{n-1} n$ !. Let $T=T^{n}$ be the maximal torus in $S O(2 n)$ and $N(T)$ be its normalizer. Then (i) $N(T)$ is generated by $W_{n}$ and $T$ multiplicatively and (ii) The Weyl group, $N(T) / T=W_{n}$.

1.1.1. LEMMA. Let $\phi_{k}: T \rightarrow T$ be the homomorphism which raises each coordinate of $T$ to its kth-power, i.e. $\phi_{k}\left(\left[z_{1}, \ldots, z_{n}\right]\right)=\left[z_{1}^{k}, \ldots, z_{n}^{k}\right]$. Then $\phi_{k}$ extends to a homomorphism, $\tilde{\phi}_{k}: N(T) \rightarrow N(T)$.

Proof. Define $\tilde{\phi}_{k}: N(T) \rightarrow N(T)$ by $\tilde{\phi}_{k}(w t)=w \phi_{k}(t), \forall w \in W_{n}, t \in T$. It is easily verified that $\tilde{\phi}_{k}$ is well defined, is a homomorphism and extends $\phi_{k}$. Q.E.D.

Let $\psi^{k}$ denote the Adams operation as defined in [1].

Received by the editors October 23, 1980 and, in revised form, July 10, 1981.

1980 Mathematics Subject Classification. Primary 55R25, 55M25; Secondary 55R50.

Key words and phrases. Sphere bundles, vector space bundles, degree, stable classes of vector space bundles, $K$-theory. 
1.1.2. Corollary. As an $N(T)$-module, $\psi^{k}(V)=\tilde{\phi}_{k}^{*}(V)$. In particular, $\psi^{k}$ maps an $N(T)$-module to a pure $N(T)$-module and not a virtual orce.

PROOF. It follows from the definition of $\psi^{k}(V)$ and Lemma 1.1.1 above.

1.1.3. COROLLARY. Let $P$ be a principal $N(T)$-bundle over a topological space $B$ and $E_{\xi}=P \times_{N(T)} V \rightarrow B$ define a vector-bundle $\xi$ on $B$. Then $\psi^{k}(\xi)$ is a pure vector-bundle and its total space, $E_{\psi} k_{(\xi)}=P \times_{N(T)} \tilde{\phi}_{k}^{*}(V)=P \times_{\bar{\phi}_{k}} V$.

PROOF. It follows from the definition of $\psi^{k}(\xi)$ and Corollary 1.1 .2 above.

1.1.4. LEMMA. Let $P$ be a principal $N(T)$-bundle over a topological space $B$, and $E_{\xi}=P \times_{N(T)} V \rightarrow B$ define a vector-bundle $\xi$ on $B$. Let $S(\xi)$ be the associated sphere bundle. Then there exists a fibrewise map, $f_{k}(\xi): E_{S(\xi)} \rightarrow E_{S\left(\psi^{k}(\xi)\right)}$ of degree $k^{n}$ at each fibre.

PROOF. Let $p_{k}$ : $S^{1} \rightarrow S^{1}$ be the $k$ th-power map defined by $p_{k}(z)=z^{k}, \forall z \in S^{1}$. Define $f_{k}: S(V)=S\left(V_{1}\right) * \cdots * S\left(V_{n}\right) \rightarrow S\left(V_{1}\right) * \cdots * S\left(V_{n}\right)=S\left(\phi_{k}^{*}(V)\right)$ be the $n$-fold join, $f_{k}=p_{k} * \cdots * p_{k}$. Then (i) $f_{k}$ is equivariant under the $N(T)$-actions on $S(V)$ and $S\left(\tilde{\phi}_{k}^{*}(V)\right)$ and (ii) $f_{k}$ defines a bundle map, $f_{k}(\xi): E_{S(\xi)}=P \times_{N(T)} S(V) \rightarrow$ $P \times_{N(T)}\left(\tilde{\phi}_{k}^{*}(V)\right)=E_{s\left(\psi^{k}(\xi)\right)}$, of degree $k^{n}$ at each fibre. Q.E.D.

1.1.5. REMARK. If $\xi$ has a spin reduction, i.e. if its structure group is reducible to the normalizer $N\left(T^{4 n}\right)$ of the maximal torus $T^{4 n}$ in $\operatorname{Spin}(8 n)$ and $\bar{f}_{k}(\xi): B^{\xi} \rightarrow$ $B^{\psi^{k}(\xi)}$, the map on the Thom complexes induced by $f_{k}(\xi)$ and $U_{\xi} \in \tilde{K}_{R}\left(B^{\xi}\right)$ and

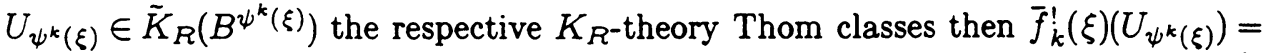
$\rho_{R}^{k}(\xi) U_{\xi}$ where $\rho_{R}^{k}$ is the characteristic class associated to the Adams operation $\psi_{R}^{k}$ via the $K_{R}$-theory Thom isomorphism as defined in [7] and [3].

The proof of this is quite straightforward and is left to the reader since it is not needed in our proof of the Adams conjecture.

1.2. Spherical Hurewicz fibrings. A fibration which is locally fibre homotopy equivalent to the product of the base space and a sphere is called a spherical Hurewicz fibring. The direct sum of two spherical Hurewicz fibrings is defined by taking joins at each fibre. The Thom complex $B^{\xi}$ of a spherical Hurewicz fibring $\xi$ over $B$ is defined by $B^{\xi}=E_{\xi \oplus 1} / s(B)$ where $s: B \rightarrow E_{\xi \oplus 1}$ is the section at infinity. If $\xi$ is a vector-bundle and $S(\xi)$ the associated sphere-bundle then the Thom complex $B^{S(\xi)}$ of $S(\xi)$ coincides with the Thom complex $B^{\xi}$ of $\xi$. We shall adopt the notation $B^{S(\xi)}$ for this space.

1.3. The transfer map $t_{p}(\xi)$. In this section we consider a slight modification of the transfer map of $[6]$. We thus rely heavily on $\S 3$ of [6] for notation and results. Let $G$ be a compact Lie group, $P$ a principal $G$-bundle over a topological space $B, F$ a compact smooth $G$-manifold and $p: E=P \times_{G} F \rightarrow B$, the associated fibre bundle over $B$ with fibre $F$. We imbed $F$ equivariantly in a representation module $V$ of $G$. We then have as in 3.5 of [6] an equivariant map $\gamma: S^{V} \rightarrow F^{+} \wedge S^{V}$ and as in 3.6 of [6] an ex-map $\gamma^{\prime}=1 \times_{G} \gamma: P \times_{G} S^{V} \rightarrow P \times_{G}\left(F^{+} \wedge S^{V}\right)$. Let $E_{\eta}=P \times_{G} V \rightarrow B$ be the associated vector-bundle to $P$ with fibre $V$ and $s$ a complementary vectorbundle to $\eta$ with fibre $W$ and trivialization $\phi: E_{\eta \oplus s} \rightarrow B \times R^{s}$. Let $\xi$ be an arbitrary $S^{n-1}$-fibration over $B$. Let $\bar{\zeta}=S(\xi \oplus 1$ and $\bar{\xi}=\xi \oplus 1$.

$$
\gamma^{\prime} \wedge_{B} 1 \wedge_{B} 1:\left(P \times_{G} S^{V}\right) \wedge_{B} E_{\bar{\zeta}} \wedge_{B} E_{\bar{\xi}} \rightarrow\left(P \times_{G}\left(F^{+} \wedge S^{V}\right)\right) \wedge_{B} E_{\bar{\zeta}} \wedge_{B} E_{\bar{\xi}} .
$$


If we identif $\tilde{y} B$ to a print on each side, the resulting quotient space on the left is $B^{S(\eta \oplus S) \oplus \xi}$ whereas the one on the right is $E^{p^{\prime} S(\eta \oplus S) \oplus p^{\prime}(\xi)}$. Let $\sigma: B^{S(\eta \oplus S) \oplus \xi} \rightarrow$ $E^{p^{\prime} S(\eta \oplus S) \oplus p^{\prime}(\xi)}$ be the induced map. We define $t_{p}(\xi)$ as the composite

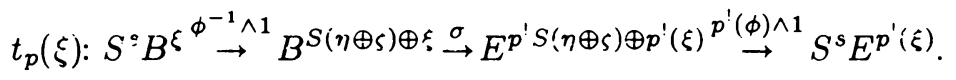

1.3.1. LEMMA. Let $U_{s \oplus \xi} \in H^{n+s}\left(S^{s} B^{\xi}\right)$ and $U_{s \oplus p^{\prime}(\xi)} \in H^{n+s}\left(S^{s} E^{p^{\prime}(\xi)}\right)$ be the cohomology Thom classe.s. Then $t_{p}^{*}(\xi)\left(U_{s \oplus p^{\prime}(\xi)}\right)=\chi(F) U_{s \oplus \xi}$.

PROOF. We have a commutative diagram

$$
\begin{aligned}
& \left(S^{n} \wedge S^{W}\right) \wedge S^{V} \stackrel{1 \wedge \gamma}{\rightarrow}\left(S^{n} \wedge S^{W}\right) \wedge\left(F^{+} \wedge S^{V}\right) \stackrel{1 \wedge \pi}{\rightarrow}\left(S^{n} \wedge S^{W}\right) \wedge S^{V} \\
& \downarrow j \quad \downarrow \quad \downarrow k \\
& S^{s} B^{\xi} \quad \stackrel{t_{p}(\xi)}{\rightarrow} \quad S^{s} E^{p^{\prime}(\xi)} \quad \stackrel{\bar{p}}{\rightarrow} \quad S^{s} B^{\xi}
\end{aligned}
$$

where $j$ and $k$ are inclusions over some point of $B$ and $\pi: F^{+} \wedge S^{V} \rightarrow S^{V}$ is the projection. Hence if $\iota \in H^{*}\left(S^{n} \wedge S^{W}\right)$ and $\iota_{V} \in H^{*}\left(S^{V}\right)$ are the generators in positive dimension then $j^{*}\left(U_{s \nsubseteq \xi}\right)=\iota \otimes \iota_{V}$ and $k^{*}\left(U_{S \otimes p^{\prime}(\xi)}\right)=\iota \otimes \iota_{V}$. By Theorem 2.6 of [6] we have $\gamma^{*} \circ \pi^{*}\left(\iota_{V}\right)=\chi(F) \iota_{V}$ and hence $(1 \wedge \gamma)^{*} \circ(1 \wedge \pi)^{*}\left(\iota \otimes \iota_{V}\right)=\chi(F)\left(\iota \otimes \iota_{V}\right)$. $t_{p}^{*}(\xi) \ddot{p}^{*}\left(U_{s \oplus \xi}\right)=m U_{s \oplus \xi}$ for some integer $m$. Thus

$$
\begin{aligned}
\chi(F)\left(\iota \otimes \iota_{V}\right) & =(1 \wedge \gamma)^{*} \circ(1 \wedge \pi)^{*}\left(\iota \otimes \iota_{V}\right)=(1 \wedge \gamma)^{*} \circ(1 \wedge \pi)^{*} \circ k^{*}\left(U_{s \oplus \xi)}\right) \\
& =j^{*} \circ t_{p}^{*}(\xi) \bar{p}^{*}\left(U_{s \oplus \xi}\right)=m j^{*}\left(U_{s \oplus \xi}\right)=m\left(\iota \otimes \iota_{V}\right) .
\end{aligned}
$$

Hence $m=\chi(F)$ and $t_{p}^{*}(\xi)\left(U_{s \oplus p^{!}(\xi)}\right)=t_{p}^{*}(\xi) \bar{p}^{*}\left(U_{s \oplus \xi_{s}}\right)=\chi(F) U_{s \oplus \xi} \quad$ Q.E.D.

1.4. The degree function $q$ on $\widetilde{K F}^{\text {or }}(B)$. Let $B$ be a finite dimensional $C W$ complex and $\widehat{K F}(B)$ the group of stable fibre homotopy classes of spherical Hurewicz fibrings over $B$ and $\widetilde{K F}^{\text {or }}(B)$ its subgroup generated by orientable fibrings. The $J$-homomorphism, $J: \tilde{K}_{R}(B) \rightarrow \widetilde{K F}(B)$, maps a vector-bundle into its associated sphere-bundle. The image of $J$ is defined to be the group $J(B)$.

Let $\xi$ and $\eta$ be $S^{m-1}$ - and $S^{n-1}$-fibrations over $B$ respectively.

1.4.1. Definition. We define $\tilde{q}(\xi, \eta)$ to be the least positive integer $\tilde{q}$ such that there exist nonnegative integers $r$ and $s$ with $m+r=n+s$ and a fibrewise map, $f: E_{\xi \oplus r} \rightarrow E_{\eta \oplus s}$, of degree $\tilde{q}$ at each fibre.

1.4.2. Definition. $q(\xi)=\tilde{q}(\xi, 0)$.

1.4.3. Translation property. $\tilde{q}(\xi, \eta)=\tilde{q}(\xi-\eta, 0)=q(\xi-\eta)$.

PROOF. Refer to $\S 4.13$ of [8].

The translation property makes clear that $\tilde{q}$ is not more general than $q$ and that it suffices to study $q$. We call $q$ the degree function on $K F^{\text {or }}(B)$. We can alternatively define $q$ using Thom complexes. Let $\xi$ be an $S^{n-1}$-fibration over $B$.

1.4.4. DEFINITION. $q(\xi)$ is the least positive integer $q$ such that there exist a nonnegative integer $r$ and a map, $f: S^{r} B^{\xi} \rightarrow S^{n+r}$, of degree $q$ (i.e. the induced cohomology homomorphism satisfies $f^{*}(\iota)=q U_{r \oplus \xi}$ where $U_{r \oplus \xi} \in H^{n+r}\left(S^{r} B^{\xi}\right)$ is the cohomology Thom class and $\iota \in H^{n+r}\left(S^{n+r}\right)$ is the generator).

Let $c_{\xi}: E_{\xi \oplus 1} \rightarrow B^{\xi}$ be the quotient projection and $c_{\xi}^{*}:\left[B^{\xi} ; S^{n}\right] \rightarrow\left[E_{\xi \oplus 1} ; S^{n}\right]$ the induced map. The equivalence of Definitions 1.4.2 and 1.4.4 follows from the existence of a map $\iota_{\xi}:\left[E_{\xi} ; S^{n-1}\right] \rightarrow\left[B^{\xi} ; S^{n}\right]$ satisfying $c_{\xi}^{*} \circ \iota_{\xi}=\Sigma$ and $\iota_{\xi \oplus 1} \circ c_{\xi}^{*}=S$ where $\Sigma$ and $S$ are the appropriate suspension maps. 
The degrees of maps $f: S^{r} B^{\xi} \rightarrow S^{n+r}$ for all $r \in Z^{+}$, form a subgroup of the integers, so $q(\xi)$ divides the degree of any such map $f$.

We note that $q$ is related to the algebraic structure by the following:

1.4.5. Nondegeneracy condition. For $u \in \widetilde{K F}^{\circ}(B), u=0$ iff $q(u)=1$. The reader is referred to [8] for details and more information on $q$.

1.5. The homomorphism, $p^{!}: \widetilde{K F}^{\text {or }}(B) \rightarrow \widetilde{K F}^{\text {or }}(E)$, for a fibre bundle $p: E \rightarrow B$. Let $F$ be a closed smooth manifold and let $p: E \rightarrow B$ define a fibre bundle with fibre $F$.

1.5.1. Proposition. If $u \in \widetilde{K F}^{\mathrm{or}}(B)$ then $q\left(p^{!}(u)\right)|q(u)| \chi(F) q\left(p^{!}(u)\right)$.

Proof. The first inequality (i.e. naturality) is obvious. The second follows from Lemma 1.3.1. For suppose $u=\xi-n$ for an $S^{n-1}$-fibration $\xi$ over $B$ and $q\left(p^{!}(u)\right)=q_{1}$. Let $t_{p}(\xi): S^{s} B^{\xi} \rightarrow S^{s} E^{p^{\prime}(\xi)}$ be the transfer map defined in $\S 1.3$. If $s$ is sufficiently large then there exists a map $f: S^{s} E^{p^{\prime}(\xi)} \rightarrow S^{n+s}$, of degree $q_{1}$. Define $g: S^{s} B^{\xi} \rightarrow S^{n+s}$ to be the composite $g=f \circ t_{p}(\xi)$. Then if $U_{s \oplus \xi} \in H^{n+s}\left(S^{s} B^{\xi}\right)$, $U_{s \oplus p^{\prime}(\xi)} \in H^{n+s}\left(S^{s} E^{p^{\prime}(\xi)}\right)$ are the cohomology Thom classes and $\iota \in H^{n+s}\left(S^{n+s}\right)$ is the generator then by Lemma 1.3.1,

$$
g^{*}(\iota)=t_{p}^{*}(\xi) \circ f^{*}(\iota)=q_{1} t_{p}^{*}(\xi)\left(U_{s \oplus p^{\prime}(\xi)}\right)=\chi(F) q_{1} U_{s \oplus \xi}
$$

Hence $g$ is of degree $\chi(F) q_{1}$ and thus $q(u) \mid \chi(F) q_{1}$. Q.E.D.

1.5.2. Corollary. If $\chi(F)=1$ then $q\left(p^{\prime}(u)\right)=q(u) \quad \forall u \in \widetilde{K F}^{\mathrm{or}}(B)$.

1.5.3 CoRollaRy. If $\chi(F)=1$ then $p ! \widetilde{K F}(B) \rightarrow \widetilde{K F}(E)$ is a monomorphism.

PROOF. It follows from Corollary 1.5.2 and the nondegeneracy of $q$.

1.6. Proof of the Adams conjecture. (i) for orientable bundles. Let $P$ be a principal $S O(2 n)$-bundle over a finite dimensional CW-complex $B, E_{\xi}=P \times S O(2 n)$ $R^{2 n}$ the total space of the associated vector-bundle $\xi$ and

$$
p: E=P / N(T) \stackrel{S O(2 n) / N(T)}{\rightarrow} P / S O(2 n)=B
$$

the projection and $E_{p^{\prime}(\xi)}=P \times_{N(T)} R^{2 n}$. By Lemma 1.1.4, there exists a fibrewise map,

$$
f_{k}\left(p^{\prime}(\xi)\right): E_{S\left(p^{\prime}(\xi)\right)} \rightarrow E_{S\left(\psi_{R}^{k}\left(p^{\prime}(\xi)\right)\right)},
$$

of degree $k^{n}$ at each fibre and hence $q\left(\left(1-\psi_{R}^{k}\right) p^{!}(\xi)\right)=\tilde{q}\left(p^{\prime}(\xi), \psi_{R}^{k}\left(p^{\prime}(\xi)\right)\right)$ divides $k^{n}$. By Corollary 1.5.2 $q\left(\left(1-\psi_{R}^{k}\right) \xi\right)=q\left(\left(1-\psi_{R}^{k}\right) p^{!}(\xi)\right)$ divides $k^{n}$. Hence by Dold's Theorem $\bmod k$ of $[2]$, the order of $J\left(\left(1-\psi_{R}^{k}\right) \xi\right)$ in $J(B)$ divides a power of $k$.

(ii) for line bundles. Let $P$ be a principal $Z_{2}$-bundle over $B, E_{\gamma}=P \times_{Z_{2}} R^{1}$ and $E_{S(c(\gamma))}=P \times z_{2} S^{1}$ be the total spaces of the associated line-bundle $\gamma$ and the sphere-bundle $S(c(\gamma))$ of its complexification $c(\gamma)$ respectively. Let $f: S^{1} \rightarrow S^{1}$ be the degree 2 map given by $f(z)=z^{2}, \forall z \in S^{1}$. Then $1 \times f: P \times S^{1} \rightarrow P \times S^{1}$, passes to the quotient and defines a bundle map $F: E_{S(c(\gamma))}=P \times_{Z_{2}} S^{1} \rightarrow\left(P / Z_{2}\right) \times S^{1}=$ $B \times S^{1}$ which is of degree 2 at each fibre. Hence by Dold's Theorem mod $k$, the order of $J(r c(\gamma))=J(2 \gamma)=2 J(\gamma)$ is a power of 2 and thus the order of $J(\gamma)$ is a power of 2 .

$$
J\left(\left(1-\psi_{R}^{k}\right) \gamma\right)= \begin{cases}J(\gamma) & \text { if } k \text { is even } \\ 0 & \text { if } k \text { is odd }\end{cases}
$$


and if $k$ is even the order of $J\left(\left(1-\psi_{R}^{k}\right) \gamma\right)$ is a power of 2 and hence divides a power of $k$. If $k$ is odd the result trivially follows.

(iii) for nonorientable bundles. Let $\eta$ be a nonorientable vector-bundle over $B$ with first Stiefel Whitney class $w_{1}(\eta)$. Let $\gamma$ be the unique line bundle over $B$ such that $w_{1}(\gamma)=w_{1}(\eta)$. Let $\xi=\eta \oplus \gamma, w_{1}(\xi)=w_{1}(\eta)+w_{1}(\gamma)=2 w_{1}(\eta)=0$ and hence $\xi$ is orientable. $J\left(\left(1-\psi_{R}^{k}\right) \eta\right)=J\left(\left(1-\psi_{R}^{k}\right) \xi\right)-J\left(\left(1-\psi_{R}^{k}\right) \gamma\right)$ and the order of the RHS divides a power of $k$ by (i) and (ii) above. Q.E.D.

2. Alternative formulations of the Adams conjecture.

2.1. Action of the Adams operations on $J(B)$. Let $J^{\prime}(B)$ be the auxiliary quotient group of $\tilde{K}_{R}(B)$ defined in [4] as follows: For $u \in \tilde{K}_{R}(B), J^{\prime}(u)=0$ iff $\rho_{R}^{k}(u)=$ $a / \psi_{R}^{k}(a), \forall k \in Z$ and for some $a \in K_{R}(B)$ of virtual dimension 1. It is a consequence of the Adams conjecture and Theorem 1.1 proved in Chapter 4 of [4] that the two groups $J(B)$ and $J^{\prime}(B)$ are isomorphic.

2.1.1. Observation. The Adams operation $\psi_{R}^{k}$ passes to the quotient and acts on the group $J(B)$.

Proof. Suppose $J(u)=0$ for $u \in \tilde{K}_{R}(B)$. Then $J^{\prime}(u)=0$ and by definition, $\rho_{R}^{k}(u)=a / \psi_{R}^{k}(a), \forall k \in Z$ and for some $a \in K_{R}(B)$ of virtual dimension 1. Applying $\psi_{R}^{s}$ to both sides we deduce that

$$
\rho_{R}^{k}\left(\psi_{R}^{s}(u)\right)=\frac{\psi_{R}^{s}(a)}{\psi_{R}^{k}\left(\psi_{R}^{s}(a)\right)}, \quad \forall k \in Z
$$

and for $\psi_{R}^{s}(a)$ of virtual dimension 1 . Thus by definition, $J^{\prime}\left(\psi_{R}^{s}(u)\right)=0$ and thus $J\left(\psi_{R}^{s}(u)\right)=0$. Q.E.D.

2.2. Preliminary lemmas.

2.2.1. Observation. Let $i=0,1,2$ or $4(\bmod 8)$ and $x \in \tilde{K}_{R}(B)$ be of filtration $\geq$ $i$. Then $\psi_{R}^{k}(x)=k^{s} x+x_{i_{2}}$ where $s=\max ([i / 2], 1), i_{2}>i$ is the least positive integer which is congruent to $0,1,2$ or $4(\bmod 8)$ and $x_{i_{2}} \in \tilde{K}_{R}(B)$ is of filtration $\geq i_{2}$.

Proof. It follows from the exact sequence of $K_{R}$-theory associated to the skeletal filtration of $B$ and the fact that $\psi_{R}^{k}=k^{s} 1$ on $\tilde{K}_{R}\left(S^{i}\right)$. Q.E.D.

2.2.2. LEMMA. Let $i=0,1,2$ or $4(\bmod 8)$ and $x \in \tilde{K}_{R}(B)$ be of filtration $\geq i$. Then for any $n \in Z^{+}$,

$$
\psi_{R}^{k^{n}}(x)=k^{n s} x+k^{(n-1) s} x_{i_{2}}+\cdots+k^{s} x_{i_{n}}+x_{i_{n+1}}
$$

where $s=\max ([i / 2], 1), i=i_{1}<i_{2}<\cdots<i_{n+1}$ is the ordered sequence of positive integers starting with $i$ and which are congruent to $0,1,2$ or $4(\bmod 8)$ and $x_{i_{j}} \in$ $\tilde{K}_{R}(B)$ is of filtration $\geq i_{j}$ for $2 \leq j \leq n+1$. Let $p$ be a prime and $J_{p}(B)$ denote the $p$-torsion of $J(B)$. If $J(x) \in J_{p}(B)$ then $J\left(x_{i_{j}}\right) \in J_{p}(B)$ for $1 \leq j \leq$ $n+1$.

Proof. By induction on $n$. It is true for $n=0$. Let $n \geq 0$ and assume it to be true for $n$. Apply $\psi_{R}^{k}$ to both sides of the equation. By Observation 2.2.1,

$$
\psi_{R}^{k}(x)=k^{s} x+x_{i_{2}} \text { and } \psi_{R}^{k}\left(x_{i_{j}}\right)=k^{\left[i_{j} / 2\right]} x_{i_{j}}+\bar{x}_{i_{j+1}} \text { for } 2 \leq j \leq n+1 \text {. }
$$

Let

$$
y_{i_{j}}=\bar{x}_{i_{j}}+k^{\left(\left[i_{j} / 2\right]-s\right)} x_{i_{j}}, \quad 2 \leq j \leq n+1,
$$


and $y_{i_{n+2}}=\bar{x}_{i_{n+2}}$. Then $y_{i_{j}} \in \tilde{K}_{R}(B)$ is of filtration $\geq i_{j}, 2 \leq j \leq n+2$, and

$$
\psi_{R}^{k^{n+1}}(x)=k^{(n+1) s} x+k^{n s} y_{i_{2}}+k^{(n-1) s} y_{i_{3}}+\cdots+y_{i_{n+2}} \text {. }
$$

If $J(x) \in J_{p}(B)$ then $J\left(x_{i_{j}}\right) \in J_{p}(B)$ by the induction hypothesis and $J\left(\bar{x}_{i_{j}}\right) \in J_{p}(B)$ by their definition and hence $J\left(y_{i_{j}}\right) \in J_{p}(B), 2 \leq j \leq n+2$.

2.2.3. LEMMA. Let $i=0,1,2$ or $4(\bmod 8)$ and $x \in \tilde{K}_{R}(B)$ be of filtration $\geq i$. Then for any $n \in Z^{+}$,

$$
k^{s+\left[i_{2} / 2\right]+\cdots+\left[i_{n} / 2\right]} x=\psi_{R}^{k^{n}}\left(x_{i_{1}}\right)+\psi_{R}^{k^{n-1}}\left(x_{i_{2}}\right)+\cdots+x_{i_{n+1}}
$$

where $s=\max ([i / 2], 1)$ and $i=i_{q}<i_{2}<\cdots<i_{n+1}$ is the ordered sequence of positive integers starting with $i$ and which are congruent to $0,1,2$ or $4(\bmod 8)$ and $x_{i_{j}} \in \tilde{K}_{R}(B)$ is of filtration $\geq i_{j}, 1 \leq j \leq n+1$.

ProOF. Obvious iteration of Observation 2.2.1 as in the proof of Lemma 2.2.2.

2.2.4. COROLlaRY. Let $i=0,1,2$ or $4(\bmod 8)$ and $x \in \tilde{K}_{R}(B)$ be of filtration $\geq i$. Then for any $n \in Z^{+}$,

$$
k^{s+\left[i_{2} / 2\right]+\cdots+\left[i_{n} / 2\right]}\left(1-\psi_{R}^{k}\right) x=\left(\psi_{R}^{k^{n}}-\psi_{R}^{k^{n+1}}\right) x_{i_{1}}+\cdots+\left(1-\psi_{R}^{k}\right) x_{i_{n+1}}
$$

where $s=\max ([i / 2], 1), i=i_{1}<i_{2}<\cdots<i_{n+1}$ is the ordered sequence of positive integers starting with $i$ and which are congruent to $0,1,2$ or $4(\bmod 8)$ and $x_{i j} \in$ $\tilde{K}_{R}(B)$ is of filtration $\geq i_{j}, 1 \leq j \leq n+1$.

ProOF. Apply $\left(1-\psi_{R}^{k}\right)$ to both sides of the equation in Lemma 2.2.3.

2.3. Alternative formulations of the Adams conjecture. Using the lemmas of $\S 2.2$ we state three alternative formulations of the Adams conjecture and prove their equivalence.

2.3.1. Proposition. Let $B$ be a finite dimensional $\mathrm{CW}$-complex.

(i) $\psi_{R}^{k}$ passes to the quotient and acts on the group $J(B)$.

(ii) If $u \in J(B)$ then the sequence $\left(\psi_{R}^{k^{n}}(u)\right)_{n \geq 1}$ stabilizes i.e. $\psi_{R}^{k^{n+1}}(u)=\psi_{R}^{k^{n}}(u)$ for sufficiently large $n$.

2.3.2. Proposition. (i) $\psi_{R}^{k}$ passes to the quotient and acts on $J(B)$.

(ii) Let $p$ be a prime. The operator $\psi_{R}^{p}$ is nilpotent on $J_{p}(B)$ and is the identity on $J_{p^{\prime}}(B)$ for $p \neq p^{\prime}$.

2.3.3. Proposition. (i) $\psi_{R}^{k}$ passes to the quotient and acts on $J(B)$.

(ii) Let $p$ be a prime. The operator $\left(1-\psi_{R}^{p}\right)$ is an isomorphism on $J_{p}(B)$ whose order is a power of $p$ and is zero on $J_{p^{\prime}}(B)$ for $p \neq p^{\prime}$.

In what follows we adopt the notation that if $n$ is an integer and $p$ a prime then $v_{p}(n)$ denotes the exponent of $p$ in the prime factorization of $n$.

2.3.4. Theorem. The Adams conjecture and Propositions 2.3.1, 2.3.2 and 2.3.3 are all equivalent. 
PROOF. (i) Adams conjecture $\Rightarrow$ Proposition 2.3.2. The first part of 2.3 .2 follows from Observation 2.1.1 which is a consequence of the Adams conjecture. Let $u=$ $J(x)$ for some $x \in \tilde{K}_{R}(B)$ of filtration $\geq i$. Since $B$ is finite dimensional, there exists an integer $d$ such that $p^{d} v=0$ uniformly for $v \in J_{p}(B)$. Choose $n \geq 2 \max (d, \operatorname{dim} B)$. Since $n \geq 2 \operatorname{dim} B$, the elements, $x_{i_{\mid n / 2\}+1}}, x_{i_{\{n / 2\}+2}}, \ldots, x_{i_{n+1}}$ associated to $\psi_{R}^{p^{n}}(x)$ by Lemma 2.2 .2 are zero and hence

$$
\psi_{R}^{p^{n}}(x)=p^{n s} x+p^{(n-1) s} x_{i_{2}}+\cdots+p^{[(n+3) / 2] s} x_{i_{|n / 2|}}
$$

where $s=\max ([i / 2], 1), u=J(x) \in J_{p}(B)$ and hence by Lemma 2.2.2, $J\left(x_{i,}\right) \in$ $J_{p}(B)$ for $1 \leq j \leq[n / 2]$. Since $n \geq 2 d$ the RHS is $J$-trivial. Thus $J\left(\psi_{R}^{p^{n}}(x)\right)=$ 0 i.e. $\psi_{R}^{p^{n}}(u)=0$. Finally, let $v \in J_{p^{\prime}}(B)$ for $p \neq p^{\prime}$. Since $\left(1-\psi_{R}^{p}\right)$ is linear, $\left(1-\psi_{R}^{p}\right) v \in J_{p^{\prime}}(B)$. By the Adams conjecture $\left(1-\psi_{R}^{p}\right) v \in J_{p}(B)$. Hence $\left(1-\psi_{R}^{p}\right) v=0$. Thus $\left(1-\psi_{R}^{p}\right)=0$ on $J_{p^{\prime}}(B)$ or equivalently $\psi_{R}^{p}=1$ on $J_{p^{\prime}}(B)$.

(ii) Proposition 2.3.2 $\Rightarrow$ Proposition 2.3.3. By Proposition 2.3.2, $\psi^{p^{e}}=0$ on $J_{p}(B)$ for some $e \in Z^{+}$. Assume also that $p^{d} u=0$ uniformly for $u \in J_{p}(B)$. Choose $s=1+d+\sup _{1 \leq j \leq e-1} v_{p}(j)$. Then on $J_{p}(B)$

$$
\left(1-\psi_{R}^{p}\right)^{p^{s}}=\sum_{j=0}^{e-1}(-1)^{j}\left(\begin{array}{c}
p^{s} \\
j
\end{array}\right) \psi_{R}^{p^{j}}
$$

It follows (essentially) from Lemma 6.1 of [5] that

$$
v_{p}\left(\left(\begin{array}{c}
p^{s} \\
j
\end{array}\right)\right)=s-v_{p}(j)>d
$$

and hence all the terms on the RHS except the first one are zero. Thus $\left(1-\dot{\psi}_{R}^{p}\right)^{p^{*}}=1$ on $J_{p}(B)$ and hence $\left(1-\psi_{R}^{p}\right)$ is an isomorphism on $J_{p}(B)$. That $\left(1-\psi_{R}^{p}\right)$ is zero on $J_{p^{\prime}}(B)$ for $p \neq p^{\prime}$ follows as in the proof of (i) above.

(iii) Proposition 2.3.3 $\Rightarrow$ Proposition 2.3.1. By Proposition 2.3.3,

$$
\left(1-\psi_{R}^{p}\right)^{p^{s}}=1+\sum_{j=1}^{p^{s}-1}(-1)^{j}\left(\begin{array}{c}
p^{s} \\
j
\end{array}\right) \psi_{R}^{p^{j}}+(-1)^{p^{s}} \psi_{R}^{p^{p^{s}}}=1 .
$$

Since

$$
p \mid\left(\begin{array}{c}
p^{s} \\
j
\end{array}\right) \text { for } 1 \leq j \leq p^{s}-1
$$

we can write

$$
\sum_{j=1}^{p^{s}-1}(-1)^{j}\left(\begin{array}{c}
p^{s} \\
j
\end{array}\right) \psi_{R}^{p^{j}}=(-1)^{p^{0}-1} p T
$$

for some operator $T$ on $J_{p}(B)$. Thus $\psi_{R}^{p^{p^{s}}}=p T$ on $J_{p}(B)$ : Since $p^{d} u=0$ uniformly for $u \in J_{p}(B)$, raising both sides to their $d$ th-power yields, $\psi_{R}^{p^{d_{p^{*}}}}=p^{d} T^{d}=0$ i.e. $\psi_{R}^{p}$ is nilpotent on $J_{p}(B)$. Let $u \in J(B)$. By Proposition 2.3.2, $\left(1-\psi_{R}^{p}\right) u \in J_{p}(B)$ and thus $\left(\psi_{R}^{p^{n}}-\psi_{R}^{p^{n+1}}\right) u=\psi_{R}^{p^{n}}\left(1-\psi_{R}^{p}\right) u=0$ for sufficiently large $n$. Finally, let $k \in Z$ and $k=p_{1} p_{2} \cdots p_{t}$ for primes $p_{i}$.

$$
\begin{aligned}
\left(\psi_{R}^{k^{n}}-\psi_{R}^{k^{n+1}}\right) u= & \left(\psi_{R}^{p_{1}^{n}}-\psi_{R}^{p_{1}^{n+1}}\right) \psi_{R}^{p_{2}^{n} \cdots p_{t}^{n}}(u)+\left(\psi_{R}^{p_{2}^{n}}-\psi_{R}^{p_{2}^{n+1}}\right) \psi_{R}^{p_{1}^{n+1}} p_{3}^{n} \cdots p_{t}^{n}(u) \\
& +\cdots+\left(\psi_{R}^{p_{t}^{n}}-\psi_{R}^{p_{t}^{n+1}}\right) \psi_{R}^{p_{1}^{n+1} p_{2}^{n+1} \cdots p_{t-1}^{n+1}}(u)=0
\end{aligned}
$$


Thus $\psi_{R}^{k^{n}}(u)=\psi_{R}^{k^{n+1}}(u)$ for sufficiently large $n$.

(iv) Proposition 2.3.1 $\Rightarrow$ Adams conjecture. By Proposition 2.3.1, there exists a positive integer $N$ such that $\left(\psi_{R}^{k^{n}}-\psi_{R}^{k^{n+1}}\right) u=0$ for $n \geq N$ and $\forall u \in J(B)$. Let $x \in \tilde{K}_{R}(B)$ be of filtration $\geq i, s=\max ([i / 2], 1)$ and choose $n \geq 2 \max (N, \operatorname{dim} B)$. Since $n \geq 2 \operatorname{dim} B$, the elements $x_{i_{|n / 2|+1}}, \ldots, x_{i_{n+1}}$ associated to $x$ by Corollary 2.2.4 are zero. Let $e=s+\left[i_{2} / 2\right]+\cdots+\left[i_{n} / 2\right]$. By Corollary 2.2.4,

$$
\begin{aligned}
k^{e}\left(1-\psi_{R}^{k}\right) x= & \left(\psi_{R}^{k^{n}}-\psi_{R}^{k^{n+1}}\right) x_{i}+\left(\psi_{R}^{k^{n-1}}-\psi_{R}^{k^{n}}\right) x_{i_{2}} \\
& +\cdots+\left(\psi_{R}^{k^{\mid(n+3) / 2]}}-\psi_{R}^{k^{|(n+5) / 2|}}\right) x_{i_{|n / 2|}}
\end{aligned}
$$

Since $n / 2 \geq N$, the RHS is $J$-trivial. Thus $k^{e} J\left(\left(1-\psi_{R}^{k}\right) x\right)=0$ for sufficiently large e. Q.E.D.

\section{REFERENCES}

1. J. F. Adams, Vector fields on spheres, Ann of Math. 75 (1962), 603-632.

2. __ On the groups $J(X)$. I, Topology 2 (1963), 181-195.

3. __ On the groups $J(X)$. II, Topology 3 (1965), 137-172.

4. $\ldots$, On the groups $J(X)$. III, Topology 3 (1965), 193-222.

5. M. F. Atiyah and J. A. Todd, On complex Stiefel manifolds, Proc. Cambridge Philos. Soc. 56 (1960), 342-353.

6. J. C. Becker and D. H. Gottlieb, Transfer map for fibre bundles, Topology 14 (1975), 1-12.

7. R. Bott, On the KO-theory of sphere bundles, Bull. Amer. Math. Soc. 68 (1962), 395-400.

8. I. Dibag, Degree theory for spherical fibrations, Tôhoku Math. J. 34 (1982), 161-177.

9. D. Quillen, Some remarks on etale homotopy theory, Topology 7 (1968), 111-116.

10.

11. D. Sullivan, Genetics of homotopy theory and the Adams conjecture, Ann. of Math. 100 (1974), 1-79.

Department of Mathematics, Middle-East Technical University, ANkara, TURKEY 